\title{
Comprehensive Analysis of a Competing Endogenous RNA Network Identifies Seven-IncRNA Signature as a Prognostic Biomarker for Melanoma
}

OPEN ACCESS

Edited by:

Francois X. Claret,

University of Texas MD Anderson Cancer Center, United States

Reviewed by:

Shizuka Uchida,

University of Louisville, United States

Simone Anfossi,

University of Texas MD Anderson Cancer Center, United States

*Correspondence: Hongxiang Chen hongxiangchen@hotmail.com

†These authors have contributed equally to this work

Specialty section:

This article was submitted to

Cancer Molecular Targets and

Therapeutics,

a section of the journa

Frontiers in Oncology

Received: 24 May 2019 Accepted: 06 September 2019 Published: 03 October 2019

Citation:

Liu N, Liu Z, Liu X and Chen H (2019)

Comprehensive Analysis of a

Competing Endogenous RNA Network Identifies Seven-IncRNA Signature as a Prognostic Biomarker for Melanoma. Front. Oncol. 9:935 doi: 10.3389/fonc.2019.00935

\author{
Nian Liu ${ }^{1+}$, Zijian Liu ${ }^{2 \dagger}$, Xinxin Liu ${ }^{1+}$ and Hongxiang Chen ${ }^{1 *}$ \\ ${ }^{1}$ Department of Dermatology, Union Hospital, Tongji Medical College, Huazhong University of Science and Technology, \\ Wuhan, China, ${ }^{2}$ Cancer Center, Tongji Medical College, Union Hospital, Huazhong University of Science and Technology, \\ Wuhan, China
}

Long non-coding RNAs (LncRNAs) can act as competing endogenous RNA (ceRNA) involving in tumor initiation and progression. Nevertheless, the prognostic roles of IncRNAs in IncRNA-related ceRNA network of melanoma remain elusive. In this study, RNA sequence profiles were downloaded from The Cancer Genome Atlas (TCGA) database, and there were 2020 differentially expressed messenger RNAs (DEmRNAs), 438 differentially expressed IncRNAs (DElncRNAs) and 65 differentially expressed microRNAs (DEmiRNAs) between primary and metastasis melanoma patients. A ceRNA regulatory network was constructed based on the DEIncRNAs-DEmiRNAs and DEmiRNAs-DEmRNAs interactions, which contained 39 IncRNAs, 10 miRNAs, and 16 mRNAs. Furthermore, univariate and multivariate Cox regression analysis were carried out to establish a 7-IncRNA prognostic signature. Subsequently, the area under the curve (AUC) value of the receiver operating characteristic $(R O C)$ curve and the KaplanMeier risk survival analysis revealed the significant performance of this signature. Finally, pathway enrichment analyses implied that IncRNA MIR205HG and MIAT were associated with multiple cancer-related pathways, especially epidermis development and immune response. The current study provides novel insights into the IncRNA-related ceRNA network and the potential of IncRNAs to be candidate prognostic biomarkers and therapeutic targets in melanoma.

Keywords: melanoma, IncRNA, ceRNA, prognostic, MIR205HG, MIAT

\section{INTRODUCTION}

Melanoma, known as a highly aggressive skin tumor, arises through malignant transformation of melanocytes and is the leading cause of skin cancer-related deaths (1). According to the global cancer statistics carried in 2018, melanoma is on the increase with around 287,723 new cases and about 60,712 cancer deaths at an estimate (2). Despite several studies have showed surgical excision of primary melanoma (PM) with high cure rate, metastatic melanoma (MM) presents a life-threatening behavior (3). To date, a lot of work has been done in melanoma, but the underlying molecular mechanisms still remain unclear $(4,5)$. Therefore, it is vital to identify potential molecular diagnostic markers and therapeutic targets in melanoma, particularly the metastatic form. 
Long non-coding RNAs (LncRNAs) are defined as any ncRNA that is longer than 200 nucleotides in length, which was once regarded as the "dark matter" for the limited or no protein-coding capacity (6). It has been reported that lncRNAs possess the capacity to regulate microRNAs' (miRNAs) function through miRNA sponge effect (6). This miRNAregulated lncRNA mechanism is a part of the "competing endogenous RNA (ceRNA) hypothesis," which was firstly proposed by Salmena et al. (7). In the ceRNA regulatory network, lncRNAs can act as endogenous molecular sponges to regulate the expression of messenger RNAs (mRNAs) through miRNA response elements (8). A growing number of researches have confirmed that lncRNAs are involved in the occurrence and progress of cancers through a disrupt in the balance of ceRNA networks, like prostate and colorectal cancer $(9,10)$.

In the past decades, several studies have investigated the potential role of lncRNAs as diagnostic or prognostic targets in cancers, such as breast cancer (11), gastric cancer (12), lung cancer (13), and melanoma (14-16). Fifteen lncRNAs were significantly correlated with the prognosis of patients with melanoma in an integrated bioinformatics analysis (14). Moreover, Chen et al. (15) and Yang et al. (16) suggested a 4-lncRNA (HCP5, LIMD1-AS1, MIR155HG, and UNQ6494) and a 6-lncRNA (AL050303, LINC00707, LINC01324, RP11-85G21, RP4-794I6.4, and RP5-855F16) signature for predicting the prognosis of melanoma patients, respectively. However, the association of lncRNAs with the prognosis of melanoma patients still remains elusive.

In the present study, the Cancer Genome Atlas (TCGA) database was used to obtain gene expression profiles to identify differentially expressed (DE) mRNAs, lncRNAs, and miRNAs between in PM and MM. Based on the above DERNAs, the lncRNA-miRNA-mRNA ceRNA network was established. Subsequently, we identified 7-lncRNA model with prognostic value based on the ceRNA regulatory network and these prognostic lncRNAs have been well-explored for biological function in melanoma. This study aimed to identify skin cancerspecific lncRNAs that are involving in prognosis and progression in melanoma.

\section{MATERIALS AND METHODS}

\section{Patient Information and Data Processing}

By virtue of genomic data commons data portal provided officially by TCGA database (https://cancergenome.nih.gov/) (17), clinical information and transcriptome profiles containing mRNA, lncRNA, and miRNA, were downloaded. A total of 435 melanoma samples were included in our analysis, comprising 96 PM samples and $339 \mathrm{MM}$ samples. This study strictly adhere to the publication guidelines provided by TCGA (https:// cancergenome.nih.gov/ publications/publicationguidelines) and as our data was obtained from TCGA database (version 2019/03/26 v16.0), there was no requirement for ethics committee approval.

\section{Analysis of Differential Expression Profiles}

The RNA expression data of lncRNAs, miRNAs, and mRNAs from TCGA database were processed. DERNAs between PM and MM were analyzed using the edge R package to identify potential molecules during the development of primary and metastatic status of melanoma. And $\left|\log _{2} \mathrm{FC}\right|>1$ and false discovery rate $(\mathrm{FDR})<0.05$ were used as cut-off criteria.

\section{Construction of CeRNA Regulatory Network}

Based on base complementary pairing principle and experimental verification, some authoritative databases provided a relationship pair such as lncRNA-miRNA and miRNA-mRNA, which can be effective for us to construct a ceRNA network centered on miRNA. Firstly, we used the miRcode database (http://www.mircode.org/) (18) to predict lncRNA-miRNA interactions, which were then combined with selected miRNAs. Secondly, three databases including Targetscan (http://www. targetscan.org/) (19), miRTarBase (http://mirtarbase.mbc. nctu.edu.tw/) (20), miRDB (http://www.mirdb.org/) (21) were resorted to predict corresponding target mRNAs of miRNAs, respectively. Finally, miRNAs that interacted with both lncRNAs and mRNAs were selected to construct the ceRNA regulatory network using Cytoscape 3.6.1 visualization software (https:// cytoscape.org/) (22).

\section{Construction of Melanoma-Specific Prognostic Model}

Univariate Cox proportional hazards regression analyses were performed based on melanoma-specific DElncRNAs in the ceRNA network. The lncRNA with a $P$-value of $<0.05$ was included in the subsequent analysis. Thereafter, we constructed a prognostic predictive model and obtained a combined prognosis score system based on those DElncRNAs. The risk score was computed as follows: Risk score $=\sum \beta \mathrm{i} \times \operatorname{expRNAi}$, where expRNA is the expression level of RNA and $\beta$ is the regression coefficient derived from the multivariate Cox regression model (23).

According to this risk score formula and our identified lncRNA signature, the patients with melanoma were classified into a high- or low-risk group based on a median risk score cut-off value. Differences of overall survival (OS) were exited between the two groups. $P<0.05$ was regarded as significant unless specifically indicated. The "survival ROC" package in $\mathrm{R}$ was used to construct the time-dependent receiver operating characteristic (ROC) curves within 3, 5, and 10 years as the defining point, and assess the sensitivity and specificity of the lncRNAs signatures in predicting survival. $P<0.05$ was considered statistically significant. Kaplan-Meier analysis was used to determine the overall survival rate between groups. All of these analyses were performed in R software (version 3.5.2) (24).

\section{Function Enrichment Analysis}

Gene ontology (GO) and Kyoto Encyclopedia of Genes and Genomes (KEGG) functional enrichment analyses were performed to predict the biological function of prognostic lncRNAs using Database for Annotation, 
TABLE 1 | Top 10 up-regulated and down-regulated differentially expressed mRNAs, IncRNAs, and miRNAs between PM and MM.

\begin{tabular}{|c|c|c|c|c|c|c|c|c|c|c|c|}
\hline mRNA & $\log _{2} F C$ & $P$-value & FDR & IncRNA & $\log _{2} F C$ & $P$-value & FDR & miRNA & $\log _{2} F C$ & $P$-value & FDR \\
\hline CYP11B1 & 9.50 & $2.81 \mathrm{E}-15$ & $9.77 \mathrm{E}-14$ & LINC00200 & 6.87 & 4.86E-16 & $1.83 \mathrm{E}-14$ & hsa-mir-521-1 & 2.97 & 4.24E-05 & 0.000324746 \\
\hline OTOR & 9.41 & $2.68 \mathrm{E}-13$ & $7.56 \mathrm{E}-12$ & LINC00824 & 5.60 & 3.36E-19 & $1.65 \mathrm{E}-17$ & hsa-mir-599 & 2.89 & $1.24 \mathrm{E}-05$ & 0.000123639 \\
\hline SFTPA2 & 8.75 & $3.32 \mathrm{E}-19$ & $1.64 \mathrm{E}-17$ & AC090150.1 & 5.25 & $2.15 \mathrm{E}-08$ & 2.69E-07 & hsa-mir-1323 & 2.31 & 0.000164931 & 0.00097977 \\
\hline SFTPB & 8.43 & $1.14 \mathrm{E}-25$ & 8.00E-24 & AC112176.1 & 5.02 & 4.55E-09 & 6.50E-08 & hsa-mir-3681 & 2.31 & $9.69 \mathrm{E}-08$ & 1.86E-06 \\
\hline SCGB1A1 & 8.27 & $9.99 \mathrm{E}-17$ & 4.06E-15 & AC104051.2 & 4.75 & $1.50 \mathrm{E}-12$ & $3.85 \mathrm{E}-11$ & hsa-mir-520a & 2.18 & 0.000115439 & 0.000738515 \\
\hline SFTPA1 & 7.65 & 3.69E-12 & 8.98E-11 & AC104823.1 & 4.74 & $3.07 \mathrm{E}-11$ & $6.45 \mathrm{E}-10$ & hsa-mir-527 & 2.12 & 0.001674285 & 0.006377622 \\
\hline STATH & 7.50 & $1.95 E-11$ & $4.24 \mathrm{E}-10$ & LINC00967 & 4.64 & 1.44E-09 & 2.29E-08 & hsa-mir-153-2 & 2.10 & $2.94 \mathrm{E}-14$ & 1.13E-12 \\
\hline SCGB3A2 & 7.25 & $3.38 \mathrm{E}-15$ & $1.16 \mathrm{E}-13$ & LINC00207 & 4.63 & 5.27E-08 & 6.09E-07 & hsa-mir-372 & 2.04 & 9.19E-06 & 9.97E-05 \\
\hline CYP17A1 & 7.07 & 2.60E-26 & $1.88 \mathrm{E}-24$ & LINC01425 & 4.35 & 1.18E-09 & 1.90E-08 & hsa-mir-675 & 2.01 & 1.79E-16 & $8.12 \mathrm{E}-15$ \\
\hline CSN2 & 6.98 & $1.99 \mathrm{E}-11$ & 4.30E-10 & AC083967.1 & 4.33 & 7.64E-22 & 4.35E-20 & hsa-mir-519c & 1.91 & 0.003929176 & 0.012178004 \\
\hline C10orf99 & -6.97 & $5.50 E-53$ & $1.28 \mathrm{E}-50$ & AL049555.1 & -3.70 & $2.90 E-27$ & $2.19 E-25$ & hsa-mir-200a & -2.34 & $8.74 \mathrm{E}-32$ & 7.27E-30 \\
\hline PI3 & -7.09 & 1.63E-102 & $2.72 E-99$ & AC245041.1 & -4.13 & $3.23 E-24$ & $2.11 \mathrm{E}-22$ & hsa-mir-200b & -2.44 & $2.48 \mathrm{E}-31$ & 1.77E-29 \\
\hline S100A7 & -7.12 & $5.54 \mathrm{E}-62$ & 1.90E-59 & AL512274.1 & -4.22 & $1.88 \mathrm{E}-87$ & $2.04 E-84$ & hsa-mir-141 & -2.61 & $3.70 \mathrm{E}-32$ & $3.69 E-30$ \\
\hline KRT6C & -7.18 & $9.64 \mathrm{E}-51$ & $1.95 E-48$ & C7orf71 & -4.28 & $1.19 \mathrm{E}-47$ & $2.12 \mathrm{E}-45$ & hsa-mir-203b & -2.67 & $7.28 \mathrm{E}-15$ & $3.03 E-13$ \\
\hline LCE3E & -7.53 & 8.37E-51 & $1.73 E-48$ & FAM83A-AS1 & -4.55 & $3.66 \mathrm{E}-58$ & 1.06E-55 & hsa-mir-200c & -2.89 & $1.80 \mathrm{E}-39$ & $2.24 \mathrm{E}-37$ \\
\hline S100A7A & -7.88 & $4.78 E-58$ & 1.36E-55 & LINC02560 & -4.83 & 5.90E-69 & $2.90 E-66$ & hsa-mir-944 & -3.28 & $1.00 \mathrm{E}-46$ & $2.50 E-44$ \\
\hline WFDC12 & -7.90 & $2.74 \mathrm{E}-75$ & $1.98 \mathrm{E}-72$ & MIR205HG & -4.85 & 7.71E-32 & 7.13E-30 & hsa-mir-6510 & -3.63 & 1.68E-16 & $8.12 \mathrm{E}-15$ \\
\hline SPRR2G & -8.24 & $6.73 E-69$ & $3.24 \mathrm{E}-66$ & FAM41C & -5.42 & 4.83E-61 & $1.59 E-58$ & hsa-mir-203a & -4.00 & $2.57 \mathrm{E}-40$ & $4.28 \mathrm{E}-38$ \\
\hline DEFB4A & -8.29 & $1.54 \mathrm{E}-77$ & 1.19E-74 & AC022081.1 & -5.59 & $2.76 \mathrm{E}-36$ & $3.20 E-34$ & hsa-mir-205 & -4.37 & 4.54E-29 & $2.83 E-27$ \\
\hline KRT9 & -9.57 & 9.10E-93 & 1.09E-89 & LINC01527 & -6.35 & $9.82 \mathrm{E}-44$ & $1.51 \mathrm{E}-41$ & hsa-mir-122 & -5.41 & $6.70 \mathrm{E}-49$ & $3.35 E-46$ \\
\hline
\end{tabular}

Visualization and Integrated Discovery (DAVID) (25), a high-throughput and integrated data-mining environment. Enrichment analysis was carried out using the functional annotation chart and functional annotation clustering options, containing KEGG pathways and GO terms. $P<0.05$ was considered to indicate statistical significance.

The microenvironment cell populations-counter (MCPcounter) was used to evaluate the association between genes and immune cell populations, using genometric mean of expression of the transcriptomic markers.

\section{Statistical Analysis}

All of the statistical analyses were performed in R software (version 3.5.2). $P<0.05$ was considered statistically significant unless otherwise mentioned.

\section{RESULTS}

\section{Identification of DERNAs in Melanoma}

A total of 435 skin cutaneous melanoma samples were obtained from TCGA database after data preprocessing. Based on the screening criteria of $\left|\log _{2} \mathrm{FC}\right|>1$ and FDR $<0.05$, 2,020 DEmRNAs (1,369 up-regulated and 651 down-regulated), 438 DElncRNAs (348 up-regulated and 90 down-regulated) and 65 DEmiRNAs (48 up-regulated and 17 down-regulated) were identified between PM and MM. Among them, top 10 DEmRNAs, DElncRNAs, and DEmiRNAs were shown in Table 1.

\section{Construction of CeRNA Regulatory}

\section{Network}

To explore the targeting relationship of the DERNAs, we focused on the interaction of miRNAs with lncRNAs and mRNAs. Firstly, we explored the regulatory loops with lncRNA-miRNA in the miRcode database, and found that 39 of 438 specific DElncRNAs might target 10 of 65 specific DEmiRNAs (Table 2). Subsequently, MiRTarBase, Targetscan, and miRDB database were used to identify miRNA-targeted mRNAs, involving 9 miRNAs and 16 mRNAs (Table 3).

Finally, on account of the regulatory pairs of lncRNA-miRNA and miRNA-mRNA, a IncRNA-miRNA-mRNA ceRNA network was constructed, using Cytoscape 3.6.1 software. In total, 39 lncRNAs, 10 miRNAs, and 16 mRNAs were included in the ceRNA regulatory network, containing 65 nodes and 145 edges (Figure 1A). Furthermore, the dysregulation of the members in the ceRNA network was displayed in a heatmap (Figure 1B).

\section{Identification of a Seven-LncRNA Prognostic Signature}

To determine the association between lncRNAs and patients' outcomes, each DElncRNA in the ceRNA network was firstly submitted to univariate Cox proportional hazards regression. Fifteen DElncRNAs in the ceRNA network were identified to have a significant prognostic value $(P<0.05$; Figure 2A). Then, a predictive model was constructed based on the coefficient of the 7 lncRNAs obtained from multivariate Cox proportional hazards regression analysis: MIR205HG, LINC00200, LIFR-AS1, H19, MIAT, AC012640.1, and PLCH1AS1 $(P<0.05$; Supplementary Table 1, Figure 2B and Table 4). 
TABLE 2 | DElncRNAs-targeted melanoma-specific intersection miRNAs.

\begin{tabular}{|c|c|}
\hline IncRNA & miRNA \\
\hline MEG3 & $\begin{array}{l}\text { hsa-mir-372, hsa-mir-373, hsa-mir-141, hsa-mir-200a, } \\
\text { hsa-mir-150, hsa-mir-206, hsa-mir-205, hsa-mir-217, } \\
\text { hsa-mir-122 }\end{array}$ \\
\hline AGAP11 & $\begin{array}{l}\text { hsa-mir-372, hsa-mir-373, hsa-mir-141, hsa-mir-200a, } \\
\text { hsa-mir-150, hsa-mir-206, hsa-mir-205, hsa-mir-217 }\end{array}$ \\
\hline MIAT & $\begin{array}{l}\text { hsa-mir-372, hsa-mir-373, hsa-mir-141, hsa-mir-200a, } \\
\text { hsa-mir-150, hsa-mir-206, hsa-mir-205 }\end{array}$ \\
\hline ADAMTS9-AS2 & $\begin{array}{l}\text { hsa-mir-372, hsa-mir-373, hsa-mir-141, hsa-mir-200a, } \\
\text { hsa-mir-150, hsa-mir-205, hsa-mir-122 }\end{array}$ \\
\hline SOX2-OT & $\begin{array}{l}\text { hsa-mir-141, hsa-mir-200a, hsa-mir-206, hsa-mir-205, } \\
\text { hsa-mir-122 }\end{array}$ \\
\hline MDS2 & $\begin{array}{l}\text { hsa-mir-141, hsa-mir-200a, hsa-mir-150, hsa-mir-205, } \\
\text { hsa-mir-122 }\end{array}$ \\
\hline KIAA0087 & hsa-mir-141, hsa-mir-200a, hsa-mir-150, hsa-mir-217 \\
\hline C17orf77 & hsa-mir-372, hsa-mir-373, has-mir-150, has-mir-206 \\
\hline FAM41C & hsa-mir-141, hsa-mir-200a, hsa-mir-206, hsa-mir-205 \\
\hline MIR205HG & hsa-mir-150, hsa-mir-206, hsa-mir-205, hsa-mir-122 \\
\hline NALCN-AS1 & hsa-mir-372, hsa-mir-373, hsa-mir-150, hsa-mir-205 \\
\hline MYCNOS & hsa-mir-150, hsa-mir-205, hsa-mir-217, hsa-mir-122 \\
\hline LINC00402 & hsa-mir-141, hsa-mir-200a, hsa-mir-150, hsa-mir-217 \\
\hline LIFR-AS1 & hsa-mir-372, hsa-mir-373, hsa-mir-150, hsa-mir-206 \\
\hline LINC00322 & hsa-mir-372, hsa-mir-373, hsa-mir-150 \\
\hline C10orf91 & hsa-mir-372, hsa-mir-373, hsa-mir-122 \\
\hline MIR137HG & hsa-mir-100, hsa-mir-150, hsa-mir-217 \\
\hline PLCH1-AS1 & hsa-mir-372, hsa-mir-373, hsa-mir-217 \\
\hline ZBTB20-AS1 & hsa-mir-206, hsa-mir-205, hsa-mir-217 \\
\hline H19 & hsa-mir-141, hsa-mir-200a, hsa-mir-206 \\
\hline IGF2-AS & hsa-mir-150, hsa-mir-122 \\
\hline AC108134.1 & hsa-mir-150, hsa-mir-206 \\
\hline LINC00393 & hsa-mir-372, hsa-mir-373 \\
\hline LINC00200 & hsa-mir-150, hsa-mir-217 \\
\hline LINC00326 & hsa-mir-150, hsa-mir-205 \\
\hline LINC00460 & hsa-mir-150, hsa-mir-206 \\
\hline AC011374.1 & hsa-mir-372, hsa-mir-373 \\
\hline AC110619.1 & hsa-mir-206, hsa-mir-122 \\
\hline LINC00266-1 & hsa-mir-217 \\
\hline LINC00207 & hsa-mir-206 \\
\hline AL163952.1 & hsa-mir-205 \\
\hline C7orf71 & hsa-mir-217 \\
\hline AL161431.1 & hsa-mir-150 \\
\hline KIF25-AS1 & hsa-mir-150 \\
\hline DNM3OS & hsa-mir-150 \\
\hline AC024597.1 & hsa-mir-122 \\
\hline ADAMTS9-AS1 & hsa-mir-150 \\
\hline AP000662.1 & hsa-mir-122 \\
\hline AC012640.1 & hsa-mir-205 \\
\hline
\end{tabular}

The risk-score formula used to calculate the risk score was as follows: $\left(0.0957^{*}\right.$ MIR205HG $)+\left(-0.1016^{*}\right.$ LINC00200 $)+$ $\left(-0.0902^{*}\right.$ LIFR-AS1 $)+\left(0.1149^{*}\right.$ H19 $)+\left(-0.169^{*}\right.$ MIAT $)+$ $\left(0.0915^{*} \mathrm{AC} 012640.1\right)+\left(0.0483^{*} \mathrm{PLCH} 1-\mathrm{AS} 1\right)$. As shown in
TABLE 3 | DEmiRNAs-targeted melanoma-specific intersection mRNAs.

\begin{tabular}{ll}
\hline miRNA & mRNA \\
\hline hsa-mir-141 & STAT4, EPHA7, ELAVL2, ZEB1, PTPRD \\
hsa-mir-200a & PTPRD, ELAVL2, ZEB1, EPHA7 \\
hsa-mir-372 & MIXL1, SIK1, TMEM100, ELAVL2 \\
hsa-mir-373 & ELAVL2, MIXL1, SIK1, TMEM100 \\
hsa-mir-205 & ESRRG, ZEB1, ENPP4 \\
hsa-mir-150 & ZEB1, RNF165, MUC4 \\
hsa-mir-206 & SFRP1, GJA1 \\
hsa-mir-217 & GPC5 \\
hsa-mir-100 & KBTBD8 \\
\hline
\end{tabular}

Figure 2B, the Concordance index of this prognostic model was 0.68 .

\section{Risk Stratification and ROC Curve Analysis}

According to the median value of the prognostic risk score, patients were divided into low- and high-risk groups. The distribution of the risk score along with the corresponding overall survival data and the expression level of 7 lncRNAs in the prognostic model were plotted and shown in Figure 3. As depicted in the picture, patients with higher risk scores tended to experience a shorter overall survival time and higher death rate $(P<0.001$; Figure 3A). Additionally, we used time-dependent ROC analysis to assess the prognostic significance of 7-lncRNA. The area under the curve (AUC) value of ROC analysis for the prognostic signature was $0.702,0.753$, and 0.732 for 3 year survival, 5-year survival, and 10-year survival, respectively (Figure 3B).

Mutations of the oncogenes BRAF and NRAS are the most common genetic alterations in cutaneous melanoma and the prognostic significance of the mutations shows differently (26). In order to better evaluate the accuracy of the model, we checked the effect of BRAF and NRAS mutation or wild-type on the prognostic ability of melanoma in the same cohort. KaplanMeier analysis showed that the higher risk score was associated with a higher mortality risk in the wild type or mutant type of these two genes (Figures 3C-F).

\section{Functional Characteristics of Prognostic LncRNAs}

It is well-known that coexpression genes pairs are more likely to be functionally related (27). To elucidate the underlying biological mechanism of the prognostic 7-lncRNA, we applied Pearson correlation coefficient to calculate the coexpression relationship between the expression levels of the 7 lncRNAs and mRNAs. In order to make the function prediction more reliable, we intersected the up- and down- regulated DEmRNAs with the positively or negatively correlated coding genes, respectively. The inclusion criteria of coexpressed gene were as follows: (a) Person correlation coefficient $>0.4$, and (b) identified from DEmRNAs mentioned above. The results showed that only MIR205HG and MIAT could meet the cut-off value, possibly because of other components with low potential biological functions 
A

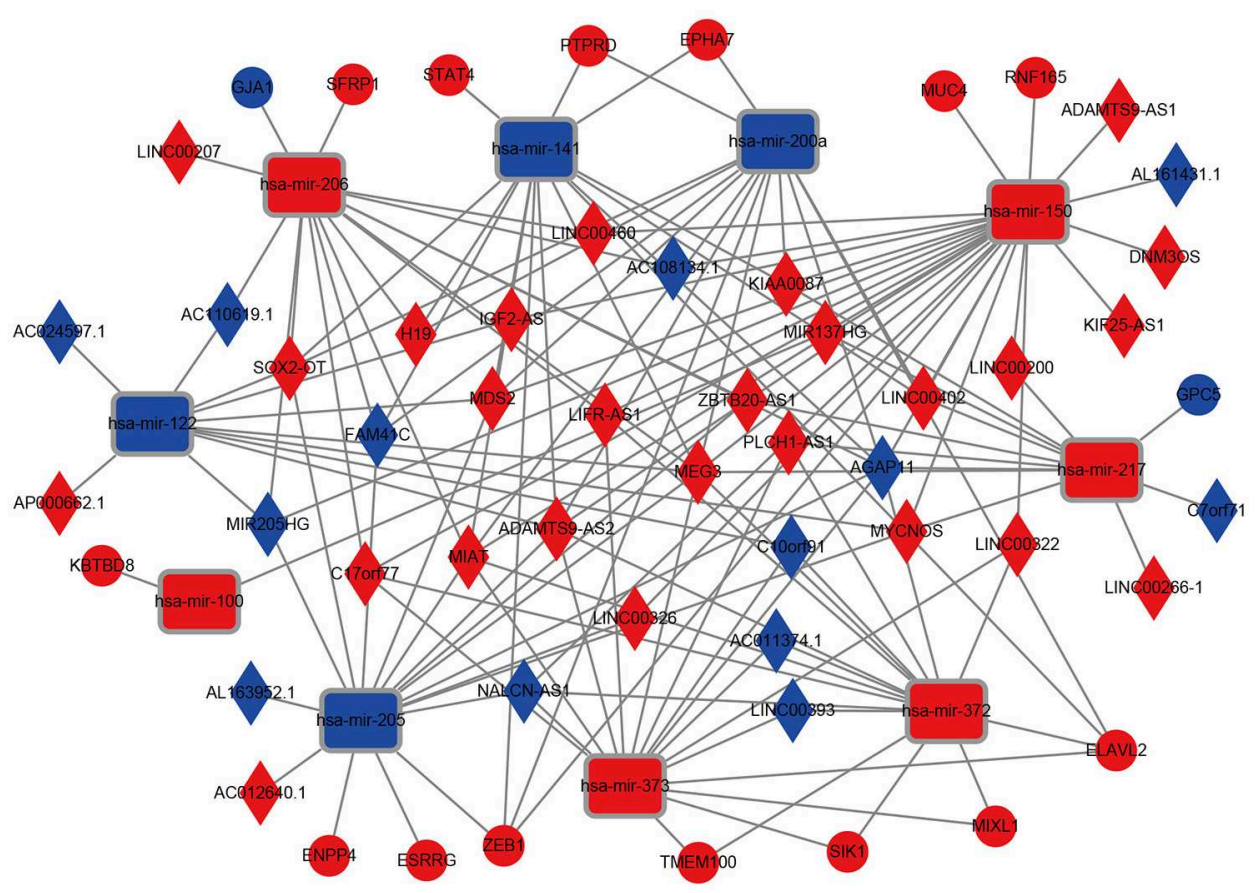

B

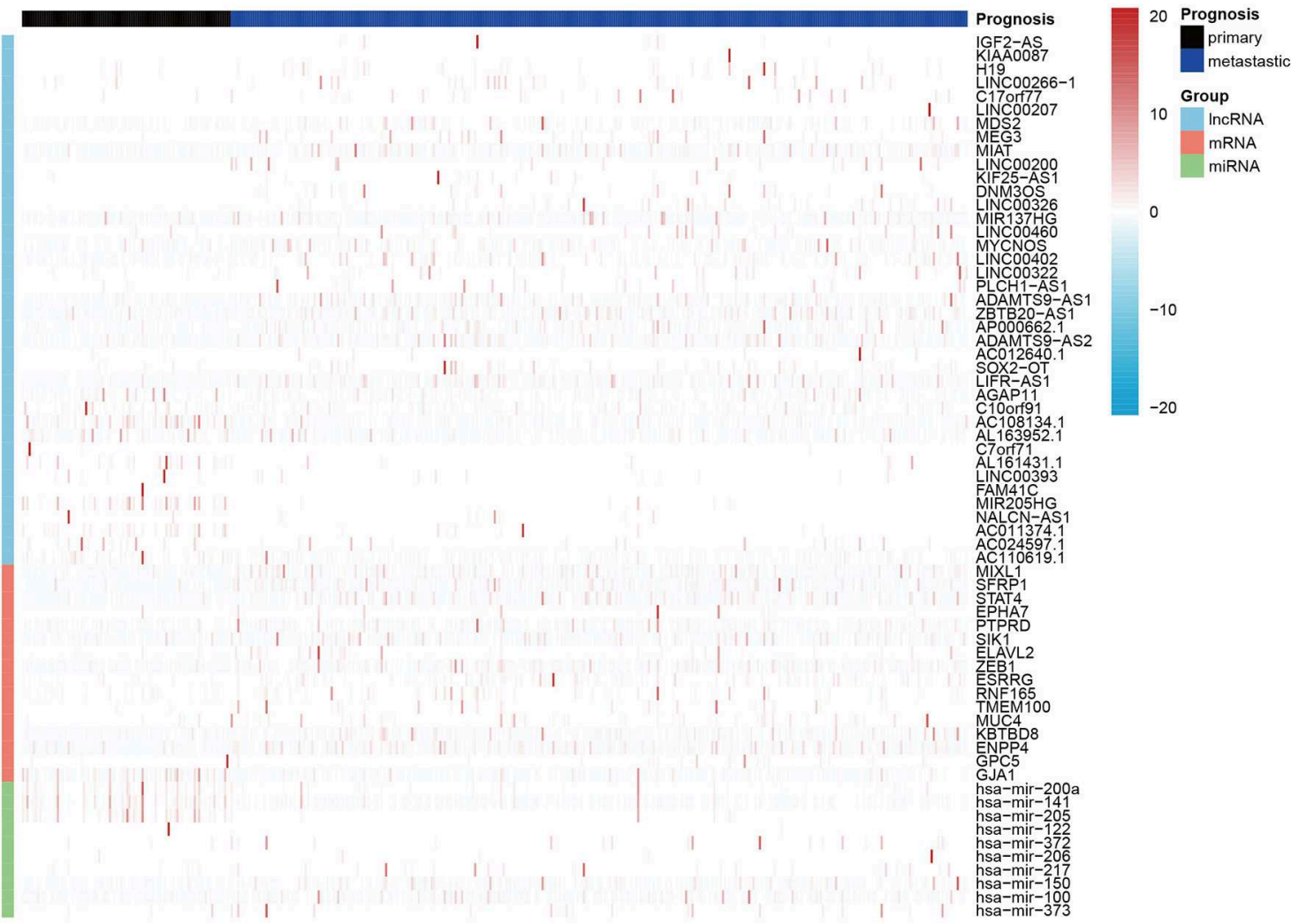

FIGURE 1 | The DERNAs in ceRNA network. (A) A global view of the ceRNA regulatory network in melanoma. Rectangles, miRNAs; rhombuses, IncRNAs; circles, mRNAs; red, up-regulation; blue, down-regulation. (B) A heatmap of DE IncRNAs, miRNAs, and mRNAs in the ceRNA network. 


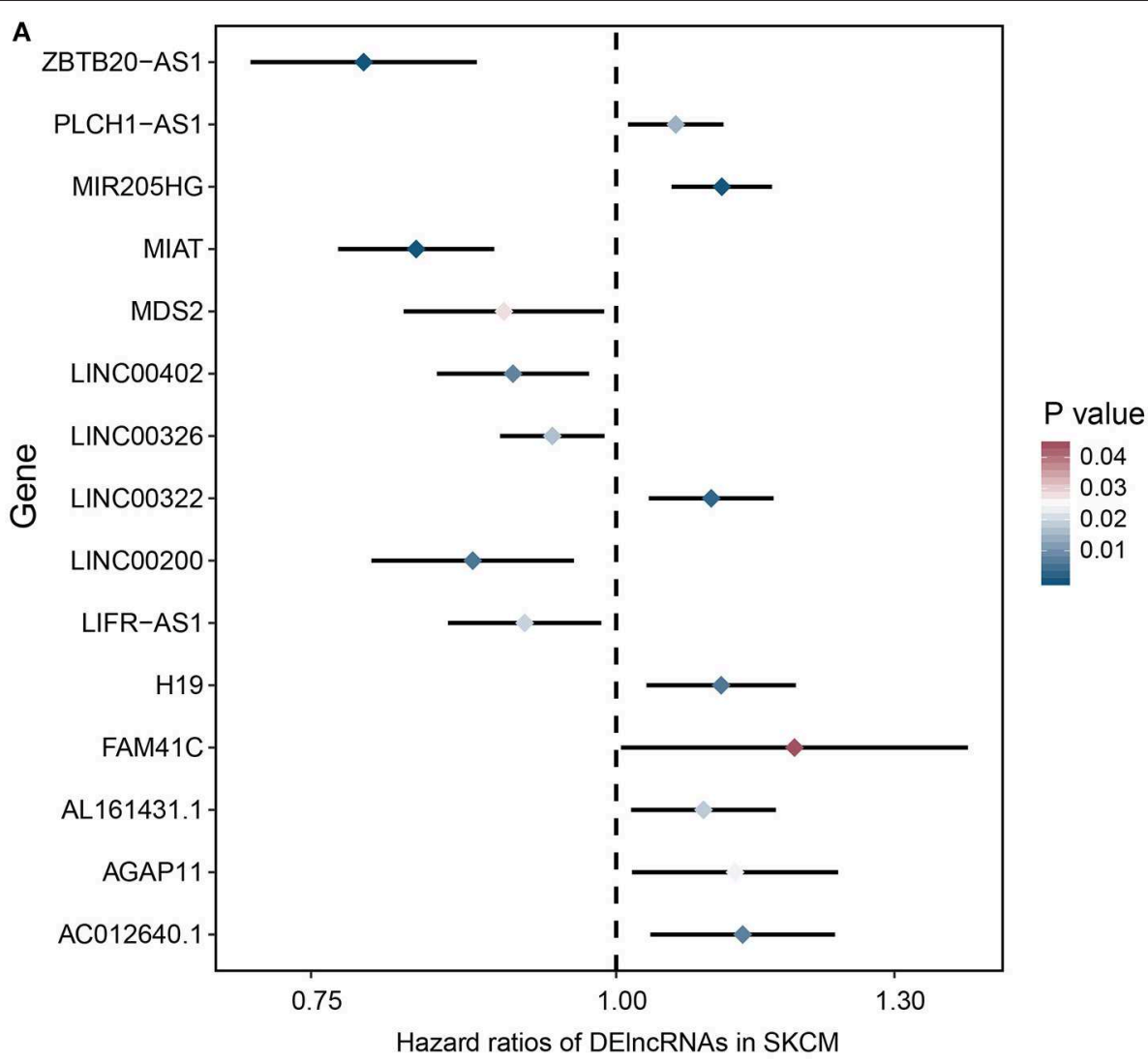

B

Hazard ratio

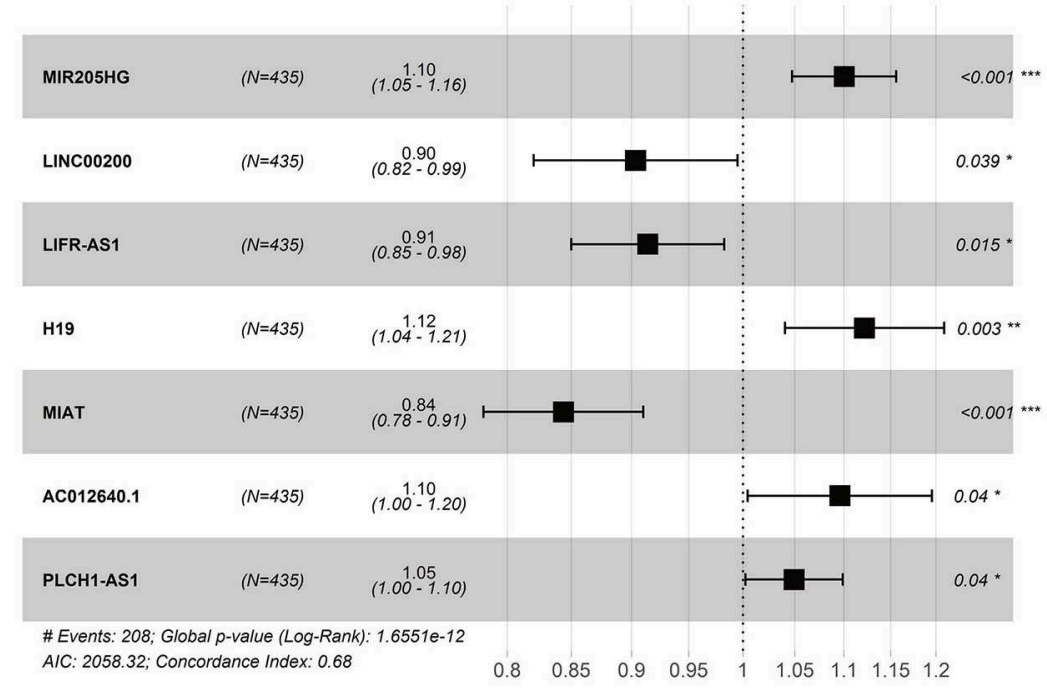

FIGURE 2 | Cox regression analyses of DE IncRNAs based on the ceRNA network. (A) 15 DElncRNAs that were significantly correlated with overall survival derived from the univariable Cox regression analysis in melanoma patients. (B) 7 DElncRNAs that were significantly correlated with overall survival derived from the multivariate Cox regression analysis in melanoma patients.

(Supplementary Figure 1). Therefore, MIR205HG and MIAT were regarded as hub lncRNAs in our prognostic model. GO and KEGG pathway enrichment analyses revealed that MIR205HG was significantly enriched for multiple pathways, including
Metabolic pathways $(P<0.05)$ and Ras signaling pathway $(P<0.05)$, as well as GO terms such epidermis development $(P<0.001$; Figures 4A,B). Moreover, MIAT was significantly enriched for multiple pathways, including Cytokine-cytokine 
TABLE 4 | Multivariate Cox regression analysis of 7 prognostic IncRNAs associated with overall survival in melanoma patients.

\begin{tabular}{lccccc}
\hline IncRNA & Coef & Exp(coef) & Se(coef) & $\mathbf{z}$ & $\boldsymbol{P}$ \\
\hline MIR205HG & 0.096 & 1.100 & 0.025 & 3.81 & 0.00014 \\
LINC00200 & -0.102 & 0.903 & 0.049 & -2.06 & 0.03904 \\
LIFR-AS1 & -0.090 & 0.914 & 0.037 & -2.44 & 0.01455 \\
H19 & 0.115 & 1.122 & 0.038 & 2.99 & 0.00278 \\
MIAT & -0.170 & 0.844 & 0.039 & -4.40 & $1.07 E-05$ \\
AC012640.1 & 0.091 & 1.096 & 0.044 & 2.06 & 0.03975 \\
PLCH1-AS1 & 0.048 & 1.050 & 0.024 & 2.06 & 0.03980 \\
\hline
\end{tabular}

receptor interaction $(P<0.001)$ and Chemokine signaling pathway $(P<0.001)$, as well as GO terms such as immune response $(P<0.001$; Figures $4 \mathrm{C}, \mathbf{D})$. To further explore the correlation between MIAT and immune response, we picked out the genes enriched in Natural killer cell mediated cytotoxicity and $\mathrm{T}$ cell receptor signaling pathway. LCK, VAV1, PTPN6, PIK3CG, ZAP70, IFNG, and CD247 were overlapped between the two signaling pathway. Based on the MCP-counter method, we also evaluated the association with MIAT, LCK, VAV1, PTPN6, PIK3CG, ZAP70, IFNG, and CD247 and immune cell populations from transcriptomic data. As shown in Figure $4 \mathrm{E}$ and Supplementary Figure 2, the fractions of different immune cells showed significantly positive correlation with the expression of MIAT and its immune related gene. And there was a strong correlation between these genes and $\mathrm{T}$ cells, $\mathrm{CD}^{+} \mathrm{T}$ cells, and NK cells.

\section{DISCUSSION}

Melanoma is known as an aggressive tumor which shows an increasing incidence and poor prognosis in the metastatic phase. The 5-year survival rate is $\sim 90 \%$ when the localized disease is detected at an early stage. However, survival rates dramatically decrease to only $5-10 \%$ for patients with distant metastasis (28). Thus, identifying novel molecular network biomarkers is needed to stratify patients for earlier detection and to improve the long-term survival rate.

LncRNAs have drawn increasing attention in tumor initiation and progression via its ceRNA activity $(29,30)$. For instance, Mou et al. (31) confirmed that the oncogenic functions of lncRNA ATB could be attributed to its ceRNA activity to enhance the expression of Yes associated protein 1 by sponging miR-590$5 \mathrm{p}$ in malignant melanoma cells. HOTAIR could exert a tumor promoting effect on malignant melanoma and act as a ceRNA suppressing miR-152-3p expression (32). Liang et al. found that lncRNA-ZFAS1 promoted the progression of melanoma through regulation of the miR-150-5p/RAB9A axis (33). LncRNA OIP5AS1 functioned as a ceRNA participating in glutamine catabolism and melanoma cells growth (34).

In the current study, a total of 2,020 DEmRNAs, 438 DElncRNAs and 65 DEmiRNAs were identified. Based on the melanoma-specific dysregulated RNAs, a ceRNA regulatory network was established, including 39 DElncRNAs,
10 DEmiRNAs, and 16 DEmRNAs. Univariate and multivariate Cox regression analyses were applied to select potential prognosis-related lncRNAs and a 7-lncRNA prognostic signature (MIR205HG, LINC00200, LIFR-AS1, H19, MIAT, AC012640.1, and PLCH1-AS1) was identified. In addition, the potential biological functions of MIR205 and MIAT were also evaluated.

With the goal of identifying lncRNAs significantly associated with OS of melanoma patients, univariate and multivariate Cox proportional regression analyses were carried out. Univariate regression analysis on the DElncRNAs of the ceRNA network identified $15 \operatorname{lncRNAs}$ that were associated with OS. Multivariate analysis showed significant prognostic value of 7 of those lncRNAs in the OS of melanoma patients. A cumulative risk score of the 7 lncRNAs was calculated, which indicated that this 7-lncRNA signature independently predicted OS in melanoma patients. And the AUC value of the ROC curve was all $>0.7$ when assessing the accuracy of the signature over 3-, 5-, and 10 -year OS rates. These results indicate that the 7 -lncRNA signature can provide a powerful prognostic tool for patients with melanoma. In addition, there is a well-known repertoire of common driver mutations in melanoma, with the most prevalent being mutations in BRAF, NRAS (26). Patients carrying mutant BRAF or NRAS always have a different prognosis comparing with those carrying wild-type genes. Thus, we reviewed the BRAF or NRAS mutation status in TCGA-SKCM dataset, and our results showed the prognostic values of the 7-lncRNA signature in melanoma patients with different subtypes.

MicroRNA-205 host gene (MIR205HG) has been found as a oncogene in cervical cancer by modulating miR-122-5p/FOXP2 axis (35). In Di et al.'s study (36), they also identified the oncogenic role of lncMIR205HG in head and neck squamous cell carcinoma. However, studies on MIR205HG remain rare, and its biological functions in the control of melanoma tumorigenesis are needed to be characterized. In our study, we identified the predictive ability of MIR205HG based on the ceRNA network, GO and KEGG pathway analyses indicated that the majority of the implicated genes were significantly involved in metabolism and epidermis development-related biological processes mediating tumor progression. Our results showed that MIR205HG might be a potential therapeutic target or candidate prognostic biomarker in melanoma.

Myocardial infarction associated transcript (MIAT) was originally identified as a candidate gene for myocardial infarction, and recently shown to participate in the progression of cancer and the process of metastasis, such as melanoma (37). For instance, Yang et al. reported the high expression of MIAT in melanoma and it promoted cell proliferation, invasion and migration through the PI3K/AKT signaling pathway (37). However, our results indicated that patients with high expression of MIAT carried out a better prognosis. In our study, pathway enrichment analysis suggested that MIAT was significantly enriched in immune response and cytokine-cytokine receptor interaction. Melanoma is characterized by high immunogenicity and is typically infiltrated by different types of immune cells. Our results also showed that the expression of MIAT and its related immune molecules were significantly associated with the infiltration of immune cells, such as $\mathrm{T}$ cells, $\mathrm{CD} 8^{+} \mathrm{T}$ cells, 
A
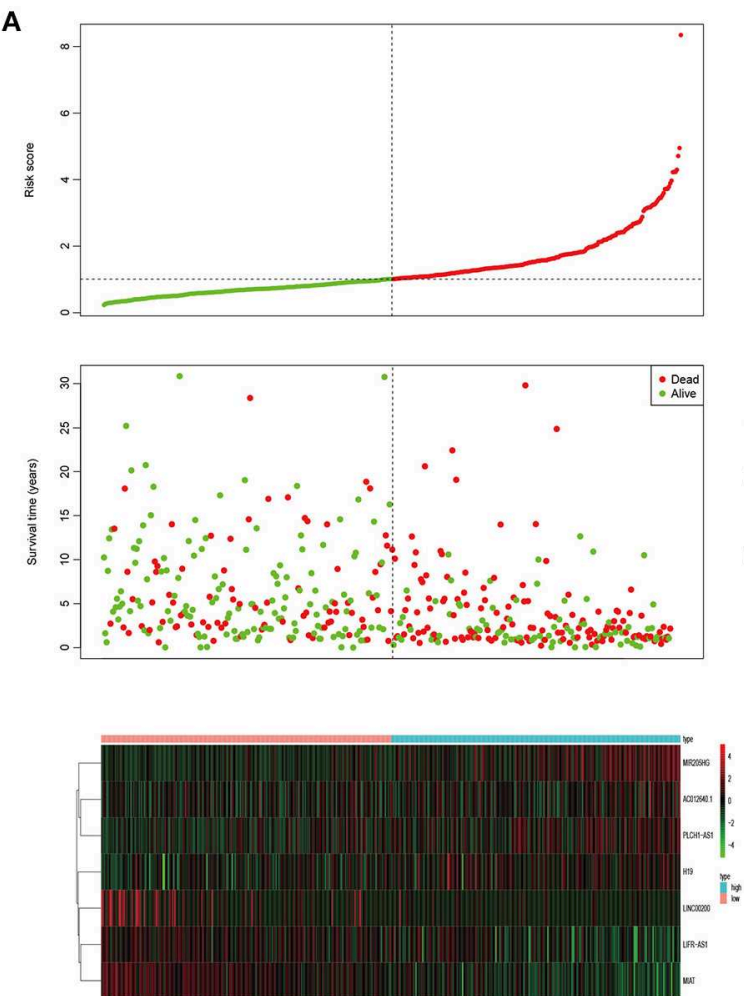

C

BRAF WT Survival curve $(p=2.062 e-09)$

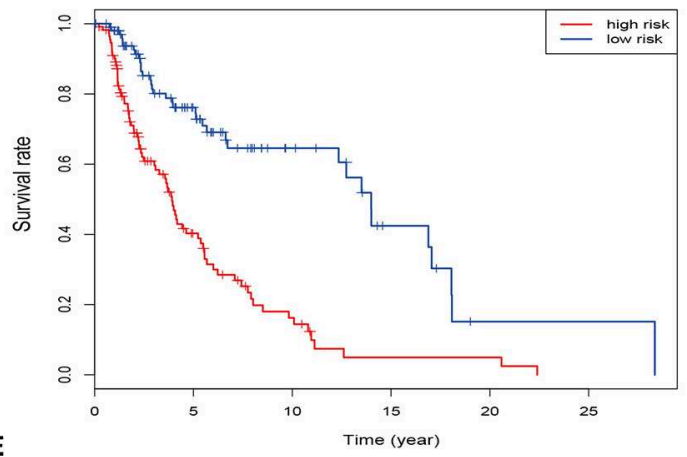

E

NRAS WT Survival curve $(p=1.164 e-10)$

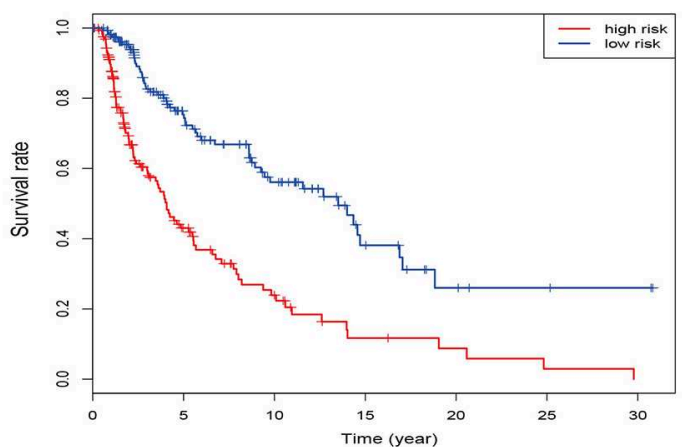

B ROC curve (3-, 5-, 10-year)

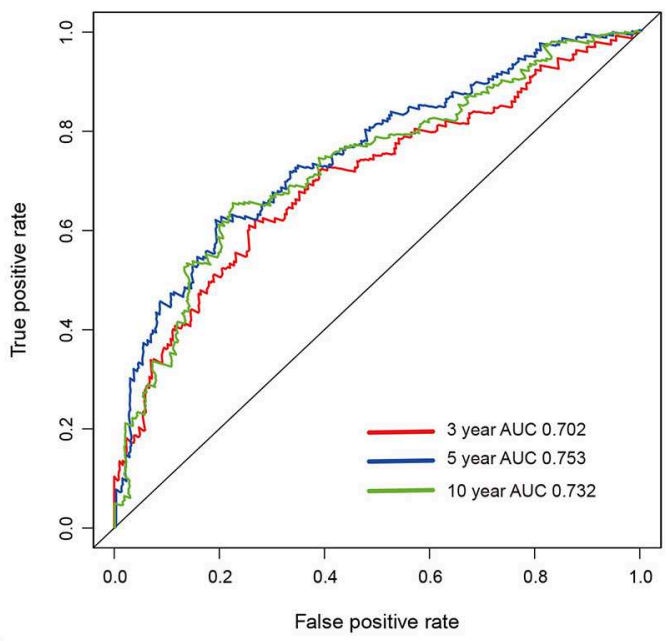

is
BRAF MT Survival curve $(p=2.349 e-07)$

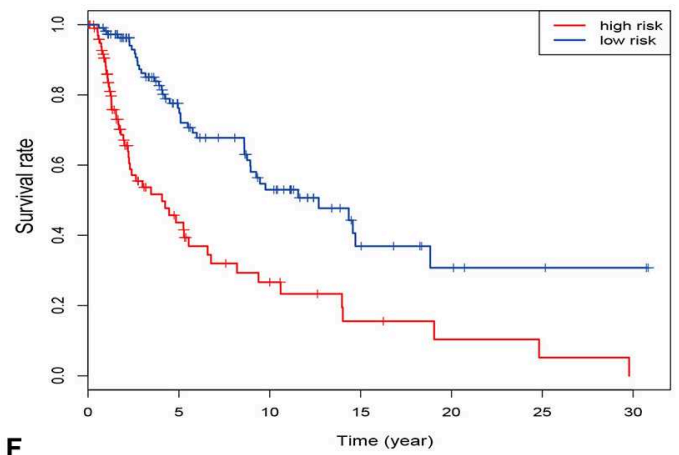

$\mathbf{F}$

NRAS MTSurvival curve $(p=3.025 e-07)$

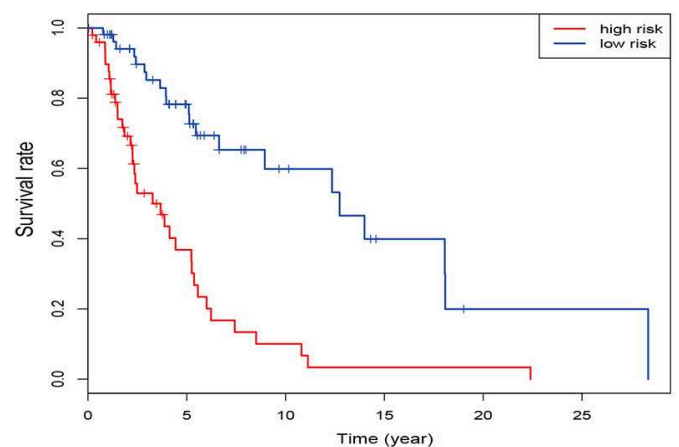

FIGURE 3 | Risk score by the 7-IncRNA signature, time-dependent ROC curve and Kaplan-Meier survival analysis. (A) The distribution of the risk score, survival status, and 7-IncRNA signature. (B) ROC curve was plotted for 3-, 5-, and 10-year overall survival in melanoma. red, 3-year; blue, 5-year; green, 10-year. (C,D) Kaplan-Meier estimates of the overall survival of patients carrying wild-type and mutant BRAF. (E,F) Kaplan-Meier analysis estimates the overall survival of patients carrying wild-type and mutant NRAS. WT, wild type; MT, mutation; red, high risk; blue, low risk. 
A

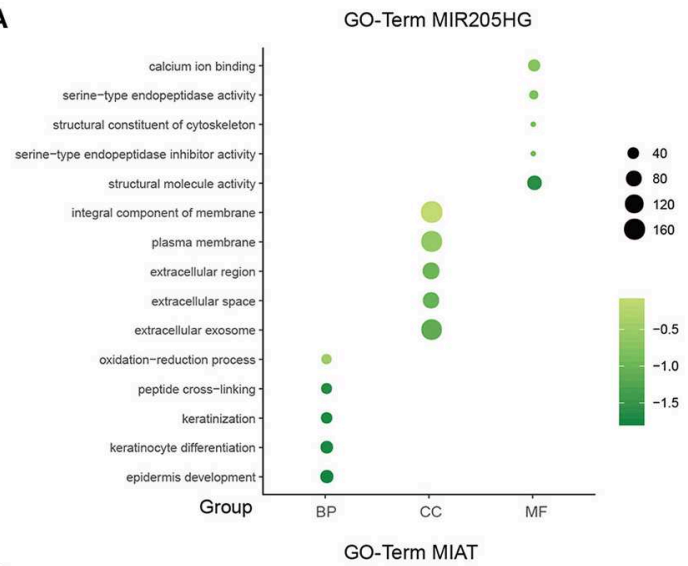

C

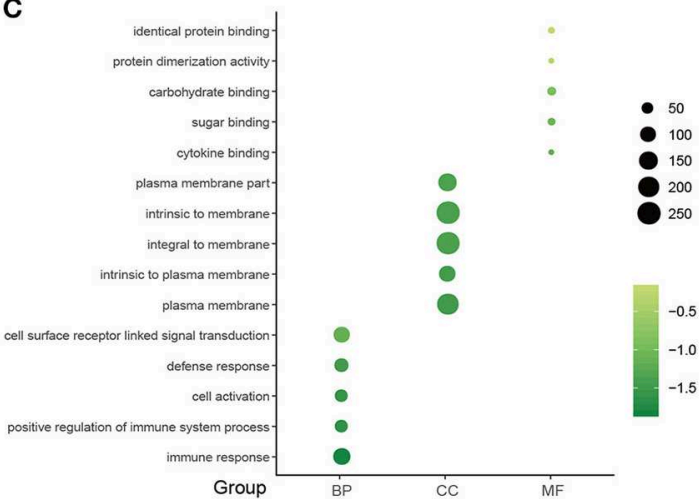

B

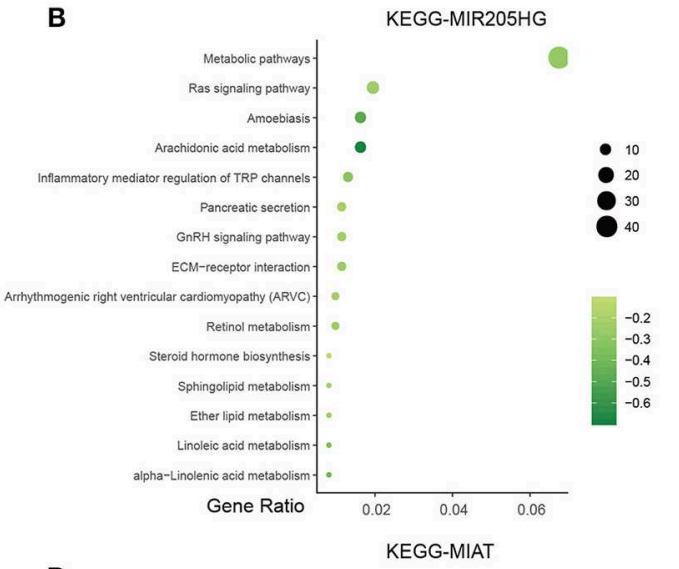

D

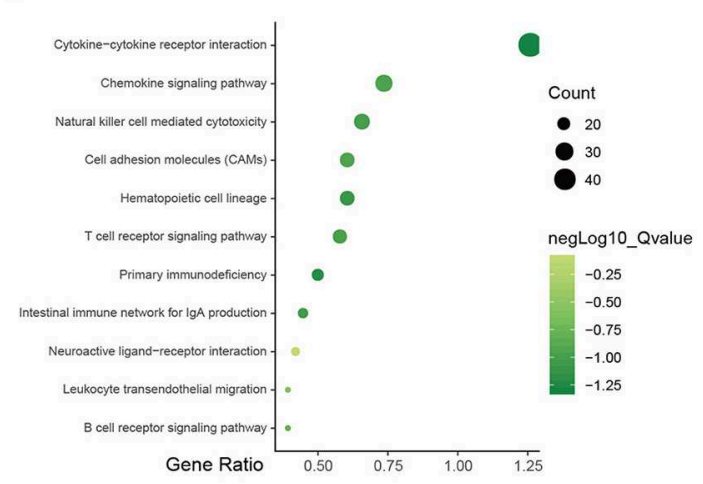

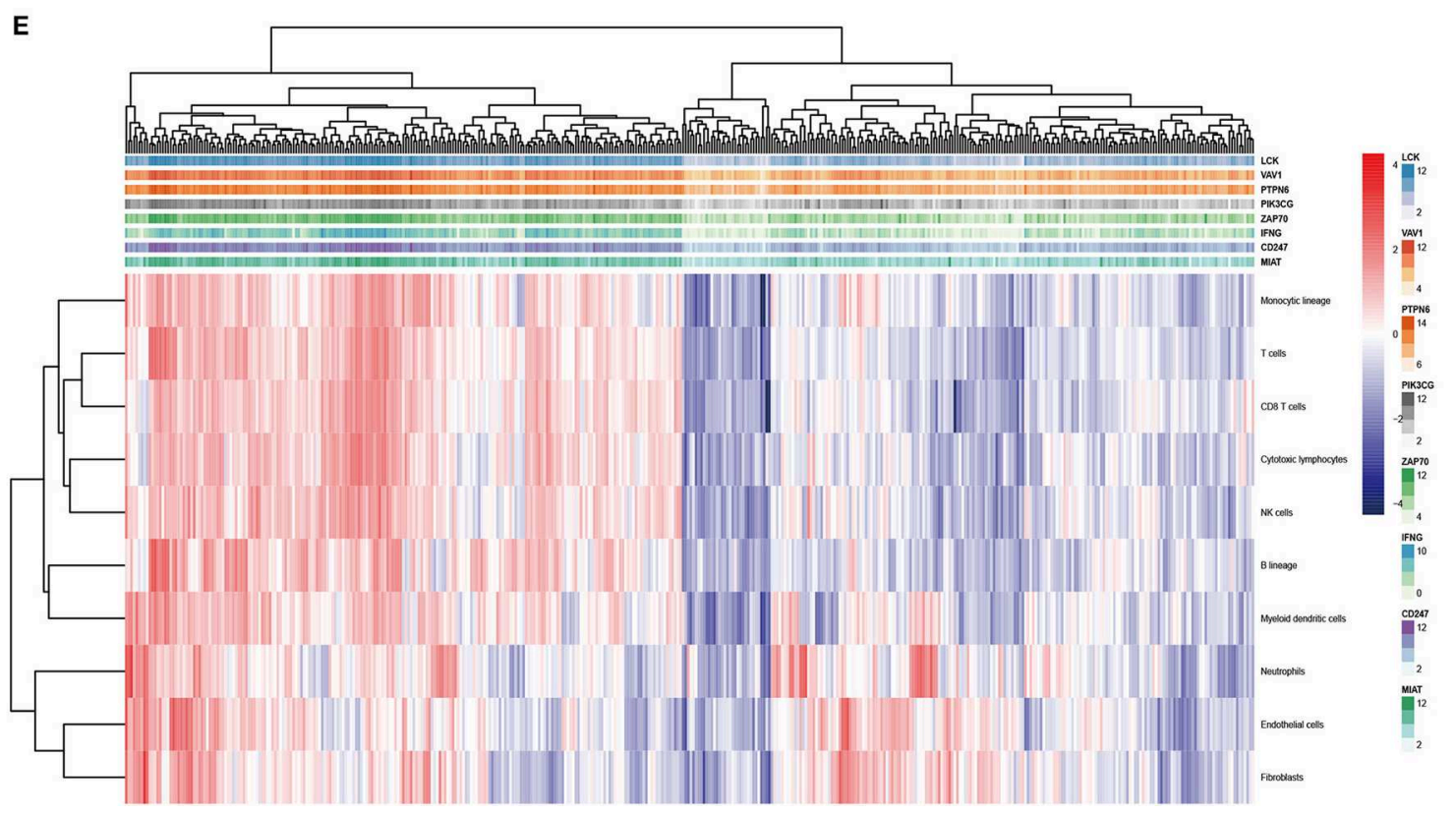

FIGURE 4 | LncRNA MIR205HG and MIAT function prediction. GO functional enrichment analysis of MIR205HG (A) and MIAT (C). X axis, GO term group; Y axis, GO terms. KEGG pathway enrichment analysis of MIR205HG (B) and MIAT (D). X axis, gene ratio; Y axis, KEGG pathway terms. light green, low enrichment; dark green, high enrichment. (E) Association between MIAT-mediated immune molecules expression and immune cell populations in melanoma. $X$ axis: genes; $Y$ axis: MIAT, MIAT-related immune molecules and immune cell. Red: high enrichment; blue: low enrichment. BP, biological process; CC, cellular component; MF, molecular function. 
and NK cells. We speculated that the higher the expression of MIAT, the more sensitive it may be to tumor immunotherapy. Furthermore, the potential strong correlation between MIAT and immune cells suggested the importance of this lncRNA in melanoma, meaning that such a lncRNA could affect prognosis via immune-related pathways.

Another lncRNA LIFR antisense RNA 1 (LIFR-AS1) is targeted by hsa-mir-206, hsa-mir-150, hsa-mir-372, and hsa-mir373. It was reported that LIFR-AS1 could predict the survival status of patients with clear cell kidney carcinoma. LIFR-AS1 also participated in modulating the resistance of colorectal cancer to photodynamic therapy, which functioned as a ceRNA regulatory network (38). H19 is a long non-coding RNA, and its abnormal expression has been found in various tumors, including melanoma. In current study, H19 is dysregulated between PM and MM. Another study also demonstrated that lncRNA H19 predicts poor prognosis in patients with melanoma and regulates cell growth, invasion, migration, and epithelialmesenchymal transition (EMT) in melanoma cells (39). And the oncogenic role of $\mathrm{H} 19$ was revealed in patients with papillary thyroid carcinoma (40). No study so far has reported any association of LINC00200, AC012640.1, and PLCH1-AS1 with cancer.

As far as we can see, it is the first report integrating a ceRNA network to build a IncRNA-related risk score and evaluate the OS of melanoma patients between PM and MM. This new approach can elucidate the lncRNA-mediated ceRNA regulatory mechanisms in the progression and prognosis of melanoma, and identify novel lncRNAs as potential diagnostic biomarkers or therapeutic targets. Compared to the previous studies $(15,16)$, our study obtained DElncRNAs between PM and MM rather than stage I-IV. We further explored the relationship between the prognostic model and the common BRAF and NRAS mutant in melanoma.

Although the findings of our study have important clinical implications, the certain limitations must also be noted. First, the prognostic value of 7-lncRNA signature remains to be verified in future studies. Second, previous works have reported some

\section{REFERENCES}

1. Tracey EH, Vij A. Updates in melanoma. Dermatol Clin. (2019) 37:7382. doi: 10.1016/j.det.2018.08.003

2. Bray F, Ferlay J, Soerjomataram I, Siegel RL, Torre LA, Jemal, A. Global cancer statistics 2018: GLOBOCAN estimates of incidence and mortality worldwide for 36 cancers in 185 countries. Cancer J Clin. (2018) 68:394424. doi: $10.3322 /$ caac. 21492

3. Schadendorf D, van Akkooi ACJ, Berking C, Griewank KG, Gutzmer R, Hauschild A, et al. Melanoma. Lancet. (2018) 392:971-84. doi: 10.1016/S0140-6736(18)31559-9

4. Wang S, Fan W, Wan B, Tu M, Jin F, Liu F, et al. Characterization of long noncoding RNA and messenger RNA signatures in melanoma tumorigenesis and metastasis. PLoS ONE. (2017) 12:e0172498. doi: 10.1371/journal.pone.0172498

5. Wang HZ, Wang F, Chen PF, Zhang M, Yu MX, Wang HL, et al. Coexpression network analysis identified that plakophilin 1 is associated with the metastasis in human melanoma. Biomed Pharmacother. (2019) 111:123442. doi: 10.1016/j.biopha.2018.12.135
lncRNAs associated with melanoma prognosis, such as DSCAMAS1, CRNDE, and CACS2, which were not involved in our results. Third, the biological roles of 7 lncRNAs in melanoma also need to be further investigated.

\section{CONCLUSION}

In summary, we have identified a 7-lncRNA signature as a potential prognostic predictor for melanoma patients by analyzing the genome-wide lncRNA expression data from the TCGA database based on a ceRNA network. The current findings provide novel insights into the lncRNA-related ceRNA network in melanoma and identify potential diagnostic and prognostic biomarkers. Further functional studies are needed to elucidate the molecular mechanisms underlying lncRNA function in melanoma.

\section{DATA AVAILABILITY STATEMENT}

Publicly available datasets were analyzed in this study. This data can be found here: https://cancergenome.nih.gov.

\section{AUTHOR CONTRIBUTIONS}

$\mathrm{NL}, \mathrm{ZL}$, and XL designed the experiments. NL and XL collected the data. NL and ZL analyzed the data. HC, NL, and ZL wrote the manuscript. HC and XL reviewed the manuscript.

\section{FUNDING}

This work was supported by the National Natural Science Foundation of China (Nos. 81673057, 81974475, and 81502730).

\section{SUPPLEMENTARY MATERIAL}

The Supplementary Material for this article can be found online at: https://www.frontiersin.org/articles/10.3389/fonc. 2019.00935/full\#supplementary-material
6. Perkel JM. Visiting "noncodarnia". Biotechniques. (2013) 54:301, 3034. doi: $10.2144 / 000114037$

7. Salmena L, Poliseno L, Tay Y, Kats L, Pandolfi PP. A ceRNA hypothesis: the Rosetta Stone of a hidden RNA language? Cell. (2011) 146:3538. doi: 10.1016/j.cell.2011.07.014

8. Pan Y, Liu G, Wang D, Li Y. Analysis of lncRNA- mediated ceRNA crosstalk and identification of prognostic signature in head and neck squamous cell carcinoma. Front Pharmacol. (2019) 10:150. doi: 10.3389/fphar.2019.00150

9. Zhang Y, Zhang D, Lv J, Wang S, Zhang Q. LncRNA SNHG15 acts as an oncogene in prostate cancer by regulating miR-338-3p/FKBP1A axis. Gene. (2019) 705:44-50. doi: 10.1016/j.gene.2019.04.033

10. Li C, Liu T, Zhang Y, Li Q, Jin LK. LncRNA- ZDHHC8P1 promotes the progression and metastasis of colorectal cancer by targeting miR-34a. Eur Rev Med Pharmacol Sci. (2019) 23:1476-86. doi: 10.26355/eurrev_201902_17105

11. Sun $\mathrm{M}, \mathrm{Wu} \mathrm{D}$, Zhou K, Li H, Gong X, Wei Q, et al. An eight-lncRNA signature predicts survival of breast cancer patients: a comprehensive study based on weighted gene co-expression network analysis and competing endogenous RNA network. Breast Cancer Res Treat. (2019) 175:5975. doi: 10.1007/s10549-019-05147-6 
12. Zhu X, Tian X, Yu C, Shen C, Yan T, Hong J, et al. A long non-coding RNA signature to improve prognosis prediction of gastric cancer. Mol Cancer. (2016) 15:60. doi: 10.1186/s12943-016-0544-0

13. Luo D, Deng B, Weng M, Luo Z, Nie X. A prognostic 4-IncRNA expression signature for lung squamous cell carcinoma. Artif Cells Nanomed Biotechnol. (2018) 46:1207-14. doi: 10.1080/21691401.2017.1366334

14. Guo L, Yao L, Jiang Y. A novel integrative approach to identify lncRNAs associated with the survival of melanoma patients. Gene. (2016) 585:21620. doi: 10.1016/j.gene.2016.03.036

15. Chen X, Guo W, Xu XJ, Su F, Wang Y, Zhang Y, et al. Melanoma long non-coding RNA signature predicts prognostic survival and directs clinical risk-specific treatments. J Dermatol Sci. (2017) 85:226-34. doi: 10.1016/j.jdermsci.2016.12.006

16. Yang $\mathrm{S}, \mathrm{Xu} J$, Zeng $\mathrm{X}$. A six-long non-coding RNA signature predicts prognosis in melanoma patients. Int J Oncol. (2018) 52:1178-88. doi: 10.3892/ijo.2018.4268

17. Weinstein JN, Collisson EA, Mills GB, Shaw KR, Ozenberger BA, Ellrott K, et al. The cancer genome atlas pan-cancer analysis project. Nat Genet. (2013) 45:1113-20. doi: 10.1038/ng.2764

18. Jeggari A, Marks DS, Larsson E. miRcode: a map of putative microRNA target sites in the long non-coding transcriptome. Bioinformatics. (2012) 28:2062-3. doi: 10.1093/bioinformatics/bts344

19. Grimson A, Farh KK, Johnston WK, Garrett-Engele P, Lim LP, Bartel DP. MicroRNA targeting specificity in mammals: determinants beyond seed pairing. Mol Cell. (2007) 27:91-105. doi: 10.1016/j.molcel.2007.06.017

20. Chou CH, Shrestha S, Yang CD, Chang NW, Lin YL, Liao KW, et al. miRTarBase update 2018: a resource for experimentally validated microRNA-target interactions. Nucleic Acids Res. (2017) 46:D296-302. doi: 10.1093/nar/gkx106

21. Wong $\mathrm{N}$, Wang $\mathrm{X}$. miRDB: an online resource for microRNA target prediction and functional annotations. Nucleic Acids Res. (2014) 43:D14652. doi: 10.1093/nar/gku1104

22. Shannon P, Markiel A, Ozier O, Baliga NS, Wang JT, Ramage $\mathrm{D}$, et al. Cytoscape: a software environment for integrated models of biomolecular interaction networks. Genome Res. (2003) 13:2498-504. doi: 10.1101/gr.1239303

23. Shukla S, Evans JR, Malik R, Feng FY, Dhanasekaran SM, Cao X, et al. Development of a RNA-Seq based prognostic signature in lung adenocarcinoma. J Natl Cancer Inst. (2016) 109:djw200. doi: 10.1093/jnci/djw200

24. Becht E, Giraldo NA, Lacroix L, Buttard B, Elarouci N, Petitprez F, et al. Estimating the population abundance of tissue-infiltrating immune and stromal cell populations using gene expression. Genome Biol. (2016) 17:218. doi: 10.1186/s13059-016-1070-5

25. Huang DW, Sherman BT, Tan Q, Collins JR, Alvord WG, Roayaei J, et al. The DAVID Gene Functional Classification Tool: a novel biological modulecentric algorithm to functionally analyze large gene lists. Genome Biol. (2007) 8:R183. doi: 10.1186/gb-2007-8-9-r183

26. Heppt MV, Siepmann T, Engel J, Schubert-Fritschle G, Eckel R, Mirlach L. Prognostic significance of BRAF and NRAS mutations in melanoma: a German study from routine care. BMC Cancer. (2017) 17:536. doi: 10.1186/s12885-017-3529-5

27. Stuart JM, Segal E, Koller D, Kim SK. A gene-coexpression network for global discovery of conserved genetic modules. Science. (2003) 302:24955. doi: 10.1126/science.1087447

28. Sun L, Guan Z, Wei S, Tan R, Li P, Yan L. Identification of long non-coding and messenger RNAs differentially expressed between primary and metastatic melanoma. Front Genet. (2019) 10:292. doi: 10.3389/fgene.2019.00292

29. Zhang H, Wang Z, Wu J, Ma R, Feng J. Long noncoding RNAs predict the survival of patients with colorectal cancer as revealed by constructing an endogenous RNA network using bioinformation analysis. Cancer Med. (2019) 8:863-73. doi: 10.1002/cam4.1813

30. Fan CN, Ma L, Liu N. Systematic analysis of lncRNA-miRNA-mRNA competing endogenous RNA network identifies four-lncRNA signature as a prognostic biomarker for breast cancer. J Transl Med. (2018) 16:264. doi: 10.1186/s12967-018-1640-2

31. Mou K, Liu B, Ding M, Mu X, Han D, Zhou Y, et al. IncRNAATB functions as a competing endogenous RNA to promote YAP1 by sponging miR-590-5p in malignant melanoma. Int J Oncol. (2018) 53:1094104. doi: 10.3892/ijo.2018.4454

32. Luan W, Li R, Liu L, Ni X, Shi Y, Xia Y, et al. Long non-coding RNA HOTAIR acts as a competing endogenous RNA to promote malignant melanoma progression by sponging miR-152-3p. Oncotarget. (2017) 8:8540114. doi: 10.18632/oncotarget.19910

33. Liang L, Zhang Z, Qin X, Gao Y, Zhao P, Liu J, et al. Long noncoding RNA ZFAS1 promotes tumorigenesis through regulation of miR-150-5p/RAB9A in melanoma. Melanoma Res. (2019). doi: 10.1097/CMR.00000000000 00595

34. Luan W, Zhang X, Ruan H, Wang J, Bu X. Long noncoding RNA OIP5AS1 acts as a competing endogenous RNA to promote glutamine catabolism and malignant melanoma growth by sponging miR-217. J Cell Physiol. (2019) 234:16609-18. doi: 10.1002/jcp.28335

35. Li Y, Wang H, Huang H. Long non-coding RNA MIR205HG function as a ceRNA to accelerate tumor growth and progression via sponging miR122-5p in cervical cancer. Biochem Biophys Res Commun. (2019) 514:7885. doi: 10.1016/j.bbrc.2019.04.102

36. Di Agostino S, Valenti F, Sacconi A, Fontemaggi G, Pallocca M, Pulito C, et al. Long non-coding MIR205HG depletes Hsa-miR-590$3 \mathrm{p}$ leading to unrestrained proliferation in head and neck squamous cell carcinoma. Theranostics. (2018) 8:1850-68. doi: 10.7150/thno. 22167

37. Yang $\mathrm{Y}$, Zhang $\mathrm{Z}$, Wu Z, Lin W, Yu M. Downregulation of the expression of the lncRNA MIAT inhibits melanoma migration and invasion through the PI3K/AKT signaling pathway. Cancer Biomark. (2019) 24:20311. doi: 10.3233/CBM-181869

38. Liu K, Yao H, Wen $\mathrm{Y}$, Zhao $\mathrm{H}$, Zhou N, Lei $\mathrm{S}$, et al. Functional role of a long non-coding RNA LIFR-AS1/miR-29a/TNFAIP3 axis in colorectal cancer resistance to pohotodynamic therapy. Biochim Biophys Acta Mol Basis Dis. (2018) 1864:2871-80. doi: 10.1016/j.bbadis.2018. 05.020

39. Shi G, Li H, Gao F, Tan Q. Incrna h19 predicts poor prognosis in patients with melanoma and regulates cell growth, invasion, migration and epithelialmesenchymal transition in melanoma cells. Onco Targets Ther. (2018) 11:3583-95. doi: 10.2147/OTT.S160143

40. Liang WQ, Zeng D, Chen CF, Sun SM, Lu XF, Peng CY, et al. Long noncoding RNA $\mathrm{H} 19$ is a critical oncogenic driver and contributes to epithelial-mesenchymal transition in papillary thyroid carcinoma. Cancer Manag Res. (2019) 11:2059-72. doi: 10.2147/CMAR.S1 95906

Conflict of Interest: The authors declare that the research was conducted in the absence of any commercial or financial relationships that could be construed as a potential conflict of interest.

Copyright (C) $2019 \mathrm{Liu}, \mathrm{Liu}, \mathrm{Liu}$ and Chen. This is an open-access article distributed under the terms of the Creative Commons Attribution License (CC BY). The use, distribution or reproduction in other forums is permitted, provided the original author(s) and the copyright owner(s) are credited and that the original publication in this journal is cited, in accordance with accepted academic practice. No use, distribution or reproduction is permitted which does not comply with these terms. 